\title{
Von den Schärfen und Krasen des Blutes
}

Zwei Jahrtausende lang hat sich die Lehre von den 4 Körpersäften als Basis von Gesundheit (Eukrasie) und Krankheit (Dyskrasie) in unserem Kulturkreis gehalten, und noch heute lebt sie in der Alternativmedizin fort. Als Begründer dieser Humoralpathologie sind Hippokrates (460-375v. Chr.) und Galen (130-200) bekannt. Die 4 Säfte Blut, gelbe und schwarze Galle sowie Schleim korrespondieren mit den 4 Elementen Feuer (warm), Luft (kalt), Erde (trocken) und Wasser (feucht). Das griechische Wort Crasis bedeutet Mischung.

Sowohl die Organpathologie des Giovanni Battista Morgagni (1682-1771) als auch die Zellularpathologie von Rudolf Virchow (1821-1902) sollten das Konzept der Humoralpathologie aus der Antike ablösen. Jedoch erlebten das 18 . und das 19. Jahrhundert eine Renaissance, wenn auch auf andere Weise („InterimsHumoralpathologie“). Anstelle der 4 Säfte kamen die Lehren der Schärfen und der Krasen, die nach den Lehrbüchern der Medizingeschichte $[1,5,16]$ vor allem mit den Namen Hyeronimus David Gaubius (1705-1780) sowie Carl Freiherr von Rokitansky (1804-1878) verbunden waren.

In der Mitte des 18. Jahrhunderts befanden sich verschiedene Lehren der Medizin in Umlauf, mit denen sich Gaubius auseinandersetzen musste. In erster Linie sind hier die Krankheitskonzepte von Friedrich Hoffmann (1660-1698), Georg Ernst Stahl (1659-1734) sowie Hermann Boerhaave (1668-1738), dem Lehrer von Gaubius, zu nennen. Vor allem jedoch konnte nicht an Morgagnis Organpathologie und an Virchows Zellularpathologie vorbeigegangen werden, in denen die festen Organe und die Zellen selbst und nicht die falsche Zusammensetzung der Fluida als hauptsächliche Ursache von Krankheiten galten [8].

\section{Die Schärfen des Blutes}

Der Münsteraner Physiologe K. E. Rothschuh erklärte die „neue Humoralpathologie“ anno 1942 folgendermaßen [14]: In der hippokratischen Säftelehre bedeutet eine richtige Mischung der vier Kardinalsäfte "Gesundheit“ (Eukrasie), eine fehlerhafte
Mischung aber „Krankheit“ (Dyskrasie). Bei der neuen Betrachtung entsteht unter der Einwirkung krankmachender Schädlichkeiten eine Krankheitsmaterie, die als Schärfe bezeichnet wird. Krankhafte Erscheinungen an den Organen werden als Folgen dieser Säfteverderbnis betrachtet. Der Organismus sucht sich von seiner Krankheit zu befreien und die gesunde Mischung der Säfte wiederherzustellen. Er sucht durch „Kochung“ (im akuten Krankheitszustand durch die Fieberhitze) die Krankheitsmaterie zu zersetzen und zur Ausscheidung reif zu machen. In der „Krisis“ wird sie in Schweiß, Harn, Blut und Stuhl aus dem Körper zur Ausscheidung gebracht.

Schärfen sind demnach Stoffwechselprodukte, die im Verlauf einer Krankheit entstehen und mit dem Blut überall hingelangen. Bei der Erkältung verursachen sie z. B. die Allgemeinbeschwerden und die Entzündung der Nasenschleimhaut oder beim Scharlach das Exanthem und die Himbeerzunge. Sie gleichen dem Begriff der Materia peccans. Bis heute sind Schärfen ein Begriff in der Naturmedizin geblieben.

\section{Hyeronimus David Gaubius}

Gaubius gehörte zu den Schülern von Hermann Boerhaave (1668-1738), der die Medizin in den Niederlanden zu einer Spitzenposition in Europa führte. Die Universität Leiden, an der Boerhaave forschte und lehrte, genoss das höchste Ansehen.

Das Hauptwerk von Gaubius, die Institutiones pathologiae medicinalis, erschien 1758 und hat die Philosophie der medizinischen Lehre über eine längere Zeitspanne bestimmt. Das Buch wurde 1792 unter dem Titel Anfangsgründe der Medicinischen Krankheitslehre ins Deutsche übersetzt [3].

\section{Der Lebensweg von H. D. Gaubius}

Die Lebensgeschichte des berühmten Arztes findet sich im Vorspann seines Buches abgedruckt [3]. Er wurde am 24. Februar 1705 in Heidelberg geboren. Sein Vater besaß mehrere Manufakturen und legte die Erziehung des Sohnes in die Hände der Jesuiten. Aber später hegte er Zweifel an der objektiven religiösen Einstellung und veranlasste die weitere Ausbildung an der berühmten Stiftung des Hallischen Waisenhaus Dr. Franke. Hier machte die strenge Frömmigkeit den jungen Hyeronimus jedoch so zuwider, dass die Schule abgebrochen wurde. Er kam zu seinem Oheim Johann Gaubius nach Amsterdam, einem bekannten Arzt. Dieser Dr. Gaubius hatte den ursprünglichen Familiennamen Gaub latinisiert. Von hier aus wurde dem Jungen der Weg zu den Vorlesungen von Boerhaave in Leiden ermöglicht; er entwickelte sich zu einem beliebten Schüler des großen Meisters und promovierte 1725 an der Universität Leiden.

Nach einer längeren Bildungsreise durch Frankreich heiratete Gaubius seine Kusine in Amsterdam. Er hatte mit ihr 6 Kinder. Von seinem Lehrer Boerhaave übernahm er zunächst das Amt eines Lektors der Chemie, bis er 1731 die Professur für Medizin und Chemie erwarb. Zu seinen besonderen Patienten gehörten die russische Kaiserin Elisabeth und Wilhelm V, Prinz von Oranien. Im hohen Lebensalter bekam er einige Gichtanfälle, welche sich immer mit der goldenen Ader endigten (Hämorrhoidalblutungen). Er starb an einem bösartigen Fieber [3].

\section{Anfangsgründe der Medicinischen Krankheitslehre}

So hieß die deutsche Übersetzung anno 1792 von Gaubius' lateinischem Werk Institutiones pathologiae medicinalis. In der Einführung zur Krankheitslehre schrieb er, Leben, Gesundheit, Krankheit und Tod machen das Ziel der menschlichen Natur auf dieser Erden und den Gegenstand der Arzneikunde aus. Im Körper ist vermöge der Mischung eine Trägheit, Beweglichkeit, Veränderlichkeit, Trieb und Uebereinstimmung, Hemmung und Schwächung zu suchen, und davon entspringen bald diese, bald jene heilsame und schädliche Ursachen [3].

Der vornehmste Grund der ganzen Arzneikunde ist in der Natur des Menschen selbst zu suchen. Durch ihre eigenen Kräfte, allein und ohne Arzt, sorgt sie für die Gesundheit der meisten Menschen, und heilt ihre Krankheiten. Die Ärzte sind Diener der Natur. Ihr sorgfältiges Studium macht den wahren und glücklichen Arzt [3]. 
Es werden einfache Krankheiten (Causa simplex) und zusammengesetzte (Composita) unterschieden. Einfache Krankheiten haben ihren Sitz in den Bestandteilen des Körpers, in den Gefäßen (Partes continentes) und in den Flüssigkeiten (Contentae). Zur Entstehung der zusammengesetzten Krankheit (Morbus compositus) treffen verschiedene einfache Zufälle zusammen, um ein Ganzes auszumachen. Die Gelegenheitsursachen aus einer dreifachen Quelle, die Seele, der Körper und die äußerlichen Dinge tragen insgesamt das ihrige. Zu letzteren gehören die Luft, die Hitze, die Kälte, Feuchtigkeit und Trockenheit, Elektrizität, Speis und Trank und viele andere [3].

Der menschliche Körper besteht, in Rücksicht auf die fühlbare Materie, aus der genauen Vermischung des Feuchten und des Trockenen. Die vier Stoffe (Erdtheil, Luft, Feuer und Wasser) sind im gesunden Zustande aufs genaueste mit einander vermischt. Es gibt Krankheiten der festen Bestandteile des Körpers (Hernien, Frakturen und Luxationen) sowie Krankheiten der Säfte. Beide Krankheitsklassen sind sehr genau miteinander verbunden. Die eine ist ohne die andere nicht denkbar, nur werden manchmal diese Säfte die Ursache, und jene die Wirkung seyn [3].

Bei den Krankheiten der Säfte gibt es die Verdünnung (Tenuitas) oder Auflösung (Dissolutio). Das Dicke (Spissitudo) entsteht durch Wassermangel. Darauf beruhen die sogenannten Schleimkrankheiten (Morbi pituitosi) mit und ohne Fieber. Bei zu starker Verdünnung der Säfte kommt es zur übermäßigen Flüssigkeitsbildung, zu einer mit Wasser verbundenen Schärfe [3].

\section{Die einzelnen Schärfen des Gaubius}

Als normale Eigenschaft der Säfte gilt die „Milde“ (Blandities), die krankhafte ist die „Schärfe“ (Acrimonia). Schärfen sind demnach krankhafte Veränderungen innerhalb des Säfteanteils des Körpers (Kasten 1). Nach ihrer Zusammensetzung lassen sich verschiedene Arten von Schärfen unterscheiden, durch ihre reizende und nagende Feuchtigkeit und durch das leichte Austreten aus den Gefäßen, Blutflüße, Durchfall, Speichelfluß, schmelzenden Urin und Schweiß, Ergießung des Blutes in das Zellengewebe, Flecken, Ausschläge, Blattern und viele andere Unordnungen im Kreislaufe in der Ernährung, Ab- und Aussonderung [3].

\section{Auswirkungen der Gaubius' schen Lehre}

Das Buch sowohl in der lateinischen Version als auch in der deutschen Übersetzung hatte eine große Ausstrahlung, weil die theoretischen Grundlagen der Krankheitslehre vor allem auf den Beobachtungen der Natur und der Bedeutung der Lebenskraft beruhen. Das bezieht auch eine subtile Beurteilung des Blutes aus dem Aderlass mit ein. Es sollte ein Lehrbuch für „die alten und neuen Ärzte“ sein [3].

Letztendlich konnten die Texte auch vom medizinischen Laien verstanden werden, wie wenige Jahre später auch die Makrobiotik des Christian Wilhelm Hufeland (1762-1836). Einzelne Krankheitssymptome, aber auch Kinder- und Volkskrankheiten werden beschrieben, jedoch nicht im Sinne der therapeutischen Konsequenz aus der Sicht des Arztes.

\section{Die Krasen}

Crasis, die Mischung, bezieht sich auf das Verhältnis der Körpersäfte zueinander. In der Theorie stehen also die Krankheiten der Dyskrasie (Humoralpathologie) den Krankheiten der Solida (Organ- und Zellularpathologie) gegenüber. Der Wiener Pathologe Carl Freiherr von Rokitansky hat in seinem Handbuch der Pathologischen Anatomie die Lehre aufgestellt, dass die Organkrankheiten mit Störungen in der Zusammensetzung des Blutes, mit „Texturkrankheiten zur Blutkrase“, beginnen. Er hat gewissermaßen beide Theorien kombiniert [12].

\section{Karl Freiherr von Rokitansky (1804-1878)}

Rokitansky wurde in Königgrätz/Böhmen geboren. Durch den frühen Tod des Vaters, eines pflichtbewussten Kreiskanzlisten, wuchs er in ärmlichen Verhältnissen auf. Er studierte in Prag und Wien. Nach den Examina heiratete er die bekannte Sängerin Anna Weiss und hatte mit ihr 4 Söhne, von denen 2 Sänger und 2 Universitätsprofessoren wurden. Schon als Student arbeitete Rokitansky in der pathologisch-anatomischen Lehranstalt des Allgemeinen Krankenhauses Wien. Hier wurde er 1844 zum ordentlichen Professor auf den ersten Lehrstuhl seines
Faches berufen. Mit ihm erreichte die Universität Wien einen Weltruf. Seine wichtigste Veröffentlichung war das Handbuch der pathologischen Anatomie anno 1855 in 3 Bänden. Es erschien in mehreren Auflagen. Als erster Pathologe löste er sich vom klinischen Bild der französischen Schule des René Théophile Hyacinthe Laennec (17811826). Er beobachtete allein die Veränderungen an der Leiche und registrierte alle Befunde ohne Voraussetzung und Befangenheit [6, 15].

Auf Rokitansky gehen der Neubau des Pathologischen Instituts im Allgemeinen Krankenhaus Wien 1862 sowie das berühmte Museum der Pathologischen Anatomie, das heute mit 49000 Objekten im Narrenturm untergebracht ist, zurück. Er war mehrmals Dekan der medizinischen Fakultät und 1852 ihr erster frei gewählter Direktor [13]. Es war die Rede vom „führenden Dreigestirn“ der Zweiten Wiener medizinischen Schule zusammen mit dem Pathologen Joseph Ritter von Skoda (1805-1881) und dem Dermatologen Ferdinand von Hebra (1816-1880) [2].

Rokitansky hat sich auch als Politiker und Philosoph betätigt. Anno 1869 wurde er zum Präsidenten der traditionsreichen Gesellschaft für Ärzte in Wien gewählt und behielt diesen Posten bis zu seinem Tode [7]. Der Verein verfügte über eine eigene Bibliothek sowie über eine eigene Zeitung, die später in die Wiener Klinische Wochenschrift überging. Im Lauf des Lebens hat Rokitansky alle Ehren und Auszeichnungen seiner Zeit erhalten.

\section{Die Krasenlehre des Carl von Rokitansky}

In der täglichen Arbeit des Pathologen, so schrieb anno 1954 der Begründer der Neurochirurgie in Wien, Leopold Schönbauer (1888-1963), gab es einen Mangel, nämlich, daß in manchen Fällen, besonders beim plötzlich eingetretenen Tode, anatomische Veränderungen nicht nachweisbar waren. Diese Tatsache war mitbestimmend für die Aufnahme der Krasenlehre in das Lehrgebäude Rokitanskys; der geringe Organbefund veranlaßte den Schluß, daß es neben den lokalisierten Krankheiten auch Allgemeinerkrankungen geben müsse. Die alte Nosologie hat beinahe nur Erkrankungen des gesamten Organismus gekannt, erst Boerhaave und Morgagni als Pathologen hatten die Auf- 
merksamkeit auf lokalisierte Prozesse gelenkt. Wo Rokitanski vom lokalen Befund nicht befriedigt war, suchte er den Krankheitssitz im Blut, das allen Körperregionen gemeinsam ist, und das er als ein Gewebe mit flüssiger Zellularsubstanz auffaßte. Er erklärte, daß Veränderungen im Blut, wie sie etwa durch Oxydationen verursacht würden, den pathologischen Zustand bedingen. Durch die Tendenz einer Allgemeinkrankheit, sich zu lokalisieren, entstehen aus einer Dyskrasie - einer fehlerhaften Mischung der Säfte - Organkrankheiten. Umgekehrt kann aber auch aus einer zunächst lokalisierten Erkrankung eine allgemeine sich entwickeln [15].

Die Krasen selbst sind das Produkt eines Blastems, unter dem sich Rokitansky eine allgemeine Ernährungsflüssigkeit, das Plasma des Blutes, vorgestellt hat, aus dem sich durch Metamorphose die Organe (Cytoblastem), aber auch Tumoren und beim Zerfallen der croupöse Faserstoff, das Tuberkel-, das Eiterblastem u.s.w. entwickeln [9, 12]. Letztendlich war das Blut ein Blastem im vollen Sinne des Wortes [9]. Das Blastem trägt das Gepräge einer dyskrastischen Mischung [12].

\section{Die Gesamtkrase}

Ohne örtliche Grundlage einer Krankheit gibt es einen primitiven dyskrastischen Prozess im Gesamtblute, eine primitive Pyämie, eine primitive croupöse Faserstoffkrase, eine Necrose (Sepsis) des Blutes usw. Diese biochemischen Veränderungen gehen der Organmanifestation einer Krankheit voraus. Aber zur Stunde hat die Chemie eine Einsicht in das Wesen nicht erlangt. Vor allem waren auch die Erreger von Infektionen nicht entdeckt. Der Pathologie kommt die Aufgabe zu, in diesem Sinne die formellen Elemente, die Blutkügelchen, und den zu einem Solidum gerinnenden Faserstoff (Thrombus) zu erforschen [12].

Die normale Krase ist präexistent, sie ist das primitive Leiden und geht der solidaren Lokalisation einer Krankheit (secundäres Leiden) voraus. Dieser Ort der Lokalisation wird durch die Beziehung der Krase mit dem Nervensystem bestimmt. Dabei spielen Hyperämie und Stase eine Rolle [12].

\section{Die einzelnen Krasen}

Eine lokale Krankheit, ein krankes Organ, sondert exsudirte Producte in die Blut- und Lymphgefässe ab. Diesen Prozess bezeich- nete Rokitanski als locale Dyskrasie (Kasten 2). Dadurch ändert sich auch die Gesamtkrase. Die Aufnahme solcher exsudierter Producte in wie immer geöffnete Blutgefäße und vor Allem der Abfluss des dyskrastische gewordenen Plasmas nach den Venen hin lokalisiert sich wiederum selbst. Ein gewisser Intensitätsgrad der lokalen Dyskrasie oder die Aufnahme einer hinreichend grossen Menge führt dann z. B. zum jauchig, septisch constituierten Plasma. Bei Lokalisation von Dyskrasien in lebensnotwendigen Organen oder gleichzeitig in vielen Organen tritt der Tod ein. Es besteht dann eine Untauglichkeit der dyskrastischen Blutmasse zur Unterhaltung lebenswichtiger Processe [12].

\section{Faserstoffige Krase}

Die Faserstoffkrase tritt in verschiedenen Formen mit Vermehrung des Faserstoffs (Hyperinose) auf. Das ist gekennzeichnet durch die Neigung zu Gerinnung innerhalb des Gefässsystems vom Herzen an bis in die Capillarität hin, und durch ihre Localisation in Entzündungen, welche im Allgemeinen sehr gefässreiche Organe - Lungen, Schleimhäute, auch seröse Häute, Zellgewebe - betreffen. Einschließlich der Pyämie entspricht die Faserstoffkrase der Dyskrasie einer Entzündung, der sog. phlogistischen Blutmischung. Von jeher wurde sie mit dem Aderlass therapiert. Die chemische Analyse hat sich bisher mit der Nachweisung des quantitativen Überschusses an Fibrin im Blute begnügt, weitere Untersuchungen sind dringend nothwendig [12].

\section{Croupöse Krase - Piorry's Haemitis}

Dieser Vorgang entspricht der Virchow'schen Thrombose. Die Krase ist durch die erhöhte Gerinnungsfähigkeit des Faserstoffs charakterisiert. Im Leben entstanden erscheinen sie (Gerinnsel, Thromben [4]) im Herzen als zerfließende sog. globulöse Vegetationen, in Gefässen größeren Calibers als cylindrisch obturirende oder als membranartige an der inneren Gefässhaut lose klebende Coagula, in capillaren Gefässen als Infarcte der Gewebe von verschiedenem Umfange. Sie sind entweder reiner Faserstoff oder sie schließen bei präcipitirter Coagulation Blutkörperchen in verschiedener Menge ein. Die Gerinnung besteht aus einem schollig faserigen Blasteme. Die Metamorphose dieser Gerinnungen besteht in einem meist ziemlich raschen Zerfallen des Blastems zu einer eiterartigen klebrigen Flüssigkeit, in welcher die Punktmasse, Kernbildungen, Zellen schwimmen [12].

\section{Tuberkel-Krase}

Die große Gruppe der Eiweißkrasen geht mit einem Mangel an Faserstoff (Hyporinose) und damit verminderter Gerinnungsneigung einher. Durch ihre engen Beziehungen zu einzelnen Organen enthalten sie spezifische Proteine [9]. Hierzu gehört auch die Tuberkelkrase. Wenn es auch ohne Zweifel völlig locale Tuberculosen gibt, so ist doch eine über das ganze Organ und noch mehr eine über mehrere Organe ausgebreitete Tuberculose immer bedingt durch eine tuberculöse Dyskrasie. Der einfachen Faserstoffkrase entspricht der graue Tuberkel. Er besteht neben einem graulichen, durchscheinenden, scholligfaserigen Blasteme aus glänzenden runden, oblongen, selbst faserig-ausgezogenen und matten, granulirten Kernen [12].

Weiter hinten führt Rokitansky aus: Jedoch reichen diese genannten Krasen keineswegs hin, das Wesen der Tuberculose genügend aufzuklären. Es muss ihnen gewiss noch eine besondere - tubeculöse - Modification ankleben [12]. Aber es dauerte noch einige Jahrzehnte bis zur Entdeckung der Tuberkelbazillen.

\section{Schärfen- und Krasenlehre in der Kritik}

Schärfen und Krasen sind Begriffe aus der „Interims-Humoralpathologie“ des 18. und 19. Jahrhunderts. Diese Epoche ist von der 2 Jahrtausende lang geltenden klassischen Lehre der vier Körpersäfte zu unterscheiden. Beide benutzen jedoch dasselbe Wort Dyskrasie gleichbedeutend mit Krankheit.

Die primären Schärfen und Krasen im Blut können zu Organkrankheiten führen. Als Entstehungsort vermutete Rokitansky dafür ein „Blastem“, das er im Interzellularraum angesiedelt sah. Andererseits verursachen aber nach Rokitanskys These auch lokalisierte Krankheiten der Organe selbst verschiedene Krasen, also Stoffwechselprodukte mit pathologischen Veränderungen des Blutes und seines Faserstoffs. Mit Erschaffung der Organpathologie des Giovanni Battista Morgagni und insbesondere 
mit der Zellularpathologie des Rudolf Virchow sollte nun die gesamte Humoralpathologie, die alte und die neue, ad absurdum geführt werden.

\section{Auseinandersetzung mit Virchow}

In einer Buchrezension griff der junge Rudolf Virchow die Krasenlehre des Carl von Rokitansky mit scharfen Worten an, obgleich er selbst als angehender Pathologe bestimmte Aspekte der Humoralpathologie zur Erklärung der Ursachen seiner Thrombose gedanklich akzeptieren musste [4]. Wegen der Einbeziehung von Krasen in das Handbuch der pathologischen Anatomie betitelte er den renommierten Autor Rokitansky als anerkannten Meister, dem sein angestammtes Gebiet zu klein geworden ist, en schrieb von Vergrößerungssucht, von Gefühl der Übermacht über die übrigen Disciplinen der Medizin [11, 17].

Dabei galt Rokitansky zur Zeit der ersten Forschungsarbeiten des jungen Virchow (anno 1843) bereits als renommierter Hochschullehrer. Seit 1834 war er als außerordentlicher Professor für Pathologie in Wien tätig. „Die armselige Baracke“ auf dem Gelände des Allgemeinen Krankenhauses Wien, wie der später in Heidelberg tätige Internist Adolf Kußmaul (1822-1902) sagte, hatte sich zu einem internationalen Zentrum der Wissenschaften entwickelt. Der Berliner Pathologe Robert Rössle (1876-1956) versuchte, die psychologischen Hintergründe des Streits zwischen Virchow und Rokitansky zu klären [11]. Er zitiert Virchows Brief an seinen Vater: In Wien ist (über das Handbuch von Rokitansky) ein großer Aufruhr ausgebrochen: Die einen, besonders die älteren Herren von der Universität und Praxis sind entzückt darüber, während die jüngeren Herren von der Wiener Schule wüten. Da ergehen nun die widersprechendsten Urteile über mich. Ich lasse es mir gefallen und denke, es wird vorübergehen und die nachhaltige Wirkung die sein, daß man künftig vorsichtiger ist.

Rössle bezeichnete Virchow fast liebevoll als „jungen Feuerkopf“ und meinte, bei aller Verschiedenheit der beiden Forschertypen gab es noch einen wesentlichen Grund für die Differenzen: Beide, Rokitansky und Virchow, standen unter dem mächtigen Einfluss der Arbeiten des organischen Chemikers Justus von Liebig (1803-1873) über den Stoffwechsel bei
Mensch und Tier, in denen auch auf die Eigenschaften des Faserstoffs eingegangen wurde. Der junge Virchow lehnte die „Hyperfibrination“ vehement ab [4]. Rokitansky hat auf Virchows Einwände nicht reagiert, aber in der 3 . Ausgabe seines Handbuchs die Krasenlehre herausgenommen [11].

Virchow hat in späteren Jahren die Genialität und die großen Verdienste von Rokitansky anerkannt. In seiner Buchbesprechung zur 3. Auflage der Pathologischen Anatomie schrieb er: Man darf es ohne weiteres als die schönste Blüte des Organismus bezeichnen, sowohl durch die meisterhafte, den Musterleistungen der beschreibenden Naturwissenschaft an die Seite zu stellende Anschaulichkeit und Genauigkeit der Schilderungen der eigenen Organkrankheiten, als auch durch die Fülle und Sicherheit der eigenen Beobachtungen. Noch bis auf den heutigen Tag ist es unerreicht geblieben $[11,18]$.

\section{Späte Rechtfertigung}

Der zu seiner Zeit recht bekannte Turiner Pathologe Benedetto Morpurgo (18611944) schrieb 1934 eine bemerkenswerte Betrachtung über die öffentlich-literarischen Dispute zur Humoralpathologie, wie sie 100 Jahre zuvor erfolgt sind. Es ist merkwürdig, daß Meinungen und Gesichtspunkte, die, während einer Periode der Entwicklung von theoretischen wissenschaftlichen Fragen (hier das System der Virchow' schen Zellularpathologie) als veraltet und überwunden erscheinen konnten, in einer späteren, mitunter weit entfernten Periode als Neuerfindungen hervortreten und für ganz moderne Ansichten gehalten werden. Einen solchen Wandel der Ideen können wir in Bezug auf die Wertung des Humoralismus und der Lehre vom amorphen Blastem verfolgen. Auf den Trümmern des Humoralismus ist vom ruhmreichen Werke Karl Rokitanskys gar nichts übrig geblieben. Jedoch wohnte der Typhuskrase, die seinerzeit als Gastroenteritis angesehen wurde, und ebenso der Tuberkelkrase ein richtiger Gedanke der allgemeinen Infektion inne. Das treue Festhalten am Humoralismus lässt Rokitansky als einen Vorgänger späterer Forschungen erscheinen, die sich auf die Entdeckungen der Hormone, der Bakterien, der Kolloide und anderer Bestandteile der leblosen Säfte beziehen [10].

\section{Abschließende Erklärung aus geschichtlicher Ferne}

Martin Müller (1878-1960), der Begründer des medizinhistorischen Instituts der Universität München, hatte den Anspruch, die Lehre von den Säften und Krasen in die theoretischen Systeme der Medizingeschichte einzuordnen [9]. Zum Ausgang des Mittelalters ist die klassische Lehre von den 4 Körpersäften und den 4 Elementen durch das Aufkommen der Chemie sinnlos geworden. William Harvey (15781657 ) hat 1628 die Vorstellungen der alten Welt durch die Entdeckung des Blutkreislaufs (De motu cordis) abgelöst. Das Blut kommt an alle Stellen des Körpers hin. Seine Zusammensetzung besteht nicht mehr aus den 4 Säften allein. Die normale Eigenschaft der Säfte ist nach Gaubius die „Milde“ (Blandities), der entgegengesetzte Fehler heißt „Schärfe“ (Acrimonia). Oder es wird mit Rokitansky gleichbedeutend von Crasis gesprochen. Krasen waren Produkte des Stoffwechsels, und man stellte sich vor, dass sie durch Oxydation von Proteinen entstehen [12]. Erst 2 Menschenalter später erbrachte die Biochemie dafür die wissenschaftlichen Grundlagen. Wir dürfen deshalb rückblickend feststellen, dass den damaligen Lehren ein wahrer Kern innewohnte.

VERSCHIEDENE SCHÄRFEN (NACH GAUBIUS)

Mechanische Schärfe (Verletzung) Chemische Schärfe (humoralische Verderbnis, Gifte)

Schärfe durch Blutungen (vielfältiger Nachtheil)

Schärfe durch Säuren (Unvermögen der Dauungskraft)

Laugenartige Schärfe (scharfe Pflanzenspeisen, Gewürze)

Fäulnis (zusammengesetzte Schärfe) Salzschärfe (Missbrauch von Küchensalz)

Salmiakschärfe (Erkrankung der Harnwege)

Spezifische Schärfen (Blasen, Blattern) 


\section{SPEZIELLE KRASEN \\ (NACH ROKITANSKY)}

1. Faserstoff-Krase

a) Einfache Faserstoffkrase

b) Croupöse Krase - Piorry's Haemitis

c) Tuberkelkrase

d) Pyämie

2. Venosität, Albuminose - Hypinosis

a) Plethora

b) Typhus-Krase

c) Exanthematische Krase

d) Hypinose bei Krht. des Nervensystems

e) Säufer-Dyskrasie

f) Krase bei der acuten Tuberculose

g) Krebs-Dyskrasie

3. Hydrämie, Anämie

a) Seröse Krase - Hydrämie

b) Anämie

4. Zersetzung - faulige, septische Krase, Sepsis

Interessenkonflikt

Die Autoren geben an, dass kein Interessenkonflikt besteht.

\section{Autorinnen/Autoren}

Wolfgang Hach

\section{Korrespondenzadresse}

Prof. Dr. Wolfgang Hach

Tacitusstr. 66

60439 Frankfurt

Deutschland

Tel.: ++ 4 97 69/57 3271

hach.frankfurt@gmx.de

\section{Literatur}

[1] Baas JH. Grundriss der Geschichte der Medizin und des heilenden Standes; Enke Stuttgart; 1876: 476

[2] Denk H. Carl Freiherr von Rokitansky. Wiener Klin Wschr 1954; 66: 129-130

[3] Gaubius HD. Anfangsgründe der Medicinischen Krankheitslehre. Aus dem Lateinischen übersetzt von Gruner CG. Voßische Buchhandlung. Berlin, 1791. Bayerische Staatsbibliothek digital am 18.10.2019. Dort auch Lebenslauf vorn. Beschreibung der Schärfen in der Mitte https://reader.digitale-sammlun gen.de/de/fs1/object/display/ bsb11107827_00005.html

[4] Hach W. Rudolf Virchow und die Erfindung der Thrombose. Gefäßchirurgie 2019; 24: 632-638

[5] Haeser H. Lehrbuch der Geschichte der Medizin und der Volkskrankheiten; Mauke; 1845: 601

[6] Hirsch A. Biografisches Lexikon der hervorragenden Aerzte aller Zeiten und Völker. Wien und Leipzig: Urban und Schwarzenberg; 1887

[7] Meister R. Carl Freiherr von Rokitansky. Präsident der Akademie der Wissenschaften. Wiener Klin Wochenschr 1954; 66 (8): 130-131

[8] Morgagni GB. De sedibus et causis morborum per anatomen indagatis. Bologna: Cornelius Haak; 1761
[9] Müller M. Rokitanskys Krasenlehre. Sudhoffs Arch Gesch Med 1930; 23: 10-39

[10] Murpurgo B. Die Lehre von Krasen und Blastem 100 Jahre nach der Antrittsvorlesung Rokitanskys in Wien. Wien Med Wschr 1834; 84: 35-36

[11] Rössle R. Karl von Rokitansky und Rudolf Virchow. Wiener Med Wschr 1934; 84 (15): 404-406

[12] Rokitansky C. Handbuch der pathologischen Anatomie. Bd 1 Wien: Braumüller und Seidel; 1846

[13] von Rokitanski C. https://de.wikipedia.org/ wiki/Gesellschaft_der_\%C3\%84rzte_in Wien\#Anf\%C3\%A4nge_der_Gesellschaft_ der \%C3\%84rzte_in_Wien. Am 20.10.2019

[14] Rothschuh KE. Zur Geschichte der Pathologie des Blutes insbesondere zur Lehre von dem Schärfen. Krasen und anderen Fehlern der Säfte. Sudhoffs Arch Gesch Med Naturwissensch 1942; 35 (5): 293-311

[15] Schönbauer L. Carl von Rokitansky. Wiener Klin Wochenschr 1954; 66 (8): 131-134

[16] Sudhoff K. Kurzes Handbuch der Geschichte der Medizin. Berlin: Karger; 1922: 311

[17] Virchow R. Stellungnahme zu Rokitansky, Handbuch der allgemeinen pathologischen Anatomie. Medicinische Zeitung 1846; 45 (49): 237-238 und 243-244 http://digi tal.zbmed.de/urn/urn:nbn:de:hbz:38m:1 85877

[18] Virchow R. Hundert Jahre Allgemeine Pathologie. Berlin: Hirschwald; 1895

\section{Bibliografie}

Phlebologie 2020; 49: 266-270

DOI 10.1055/a-1044-4584

ISSN 0939-978X

c) 2020. Thieme. All rights reserved. Georg Thieme Verlag KG, Rüdigerstraße 14, 70469 Stuttgart, Germany 